\title{
Best practices in refugee mental health
}

Elise Quint, Hasan Hawilo, Sydney Eaton

\section{ABSTRACT}

Canada has a long history of accepting immigrants and refugees. These populations may have unique health care needs that the Canadian health care system must adequately address. Currently, there are numerous barriers to the treatment of refugee mental health concerns. These include cultural, language, financial, and systemic barriers. Best practices for treatment in this population include psychotherapies such as cognitive behavioural therapy and narrative exposure therapy, medications, and health promotion and psychosocial interventions. Physicians have previously advocated for improving health care for Canada's refugee population and medical schools are working to incorporate multicultural education into their curricula.

\section{INTRODUCTION}

Worldwide conflicts have led to an increase in the number of refugees in recent years. According to the United Nations High Commissioner for Refugees, by 2017 there were 25.4 million refugees worldwide. ${ }^{1}$ The United Nations defines refugees as people who have been forced to leave their country of nationality due to fear of persecution. ${ }^{2}$ Canada has a long history of accepting refugees from as far back as 1776, and in 2015 notably pledged to accept 25000 refugees displaced after the conflict in Syria., ${ }^{3,4}$ In 2016, Canada welcomed 46700 refugees primarily from Syria, Eritrea, Iraq, Congo, and Afghanistan. ${ }^{4}$

Having accepted a large number of refugees, Canada must now find a way to meet their diverse needs, including medical needs. Estimates of the prevalence of post-traumatic stress disorder (PTSD) and depression in refugees vary, but may be as high as $30 \%{ }^{5}$ However, the prevalence of these disorders differs between refugee populations, because rates of PTSD and depression are related to the particular traumas that refugees have experienced. ${ }^{5}$ These specific factors include exposure to torture, the severity of the trauma experience, and the person's post-displacement environment. ${ }^{5}$

In this paper, we will discuss barriers to treatment of refugee mental health concerns, specific recommendations for treatment in this population, and ways that physicians and medical students can advocate for improving refugee health care.

\section{BARRIERS TO TREATMENT}

Refugees are at much higher risk of having unmet health care needs compared to the general population of Canada. In a crosssectional study of Syrian refugees conducted in 2016, $49 \%$ of refugees reported having unmet health care needs, compared to $11.2 \%$ of the general Canadian population. ${ }^{6}$
Cultural barriers. Stigma associated with mental health concerns is not a problem exclusive to Canada's refugee population; however, refugees may have unique reasons for feeling that mental health problems are stigmatized. Shannon et al. (2017) conducted focus groups in the United States with refugees from Burma, Bhutan, Somalia, and Ethiopia; these cultural groups are also represented among Canadian refugees. ${ }^{3}$ Their research found that a history of political repression, fear, a belief that "talking does not help", and avoiding symptoms were among the reasons that refugees were reluctant to discuss mental health, and were therefore less likely to seek mental health care. ${ }^{7}$ In addition, some patients may prefer a particular gender or ethnicity of care provider which cannot always be accommodated. ${ }^{8}$

Language barriers. Language barriers become critically important when related to mental health care. In many cases, health care institutions are unable or unwilling to provide formal translation services. ${ }^{9}$ Informal translation is then usually provided by the patient's friend or family member. The presence of this third person in the room with the provider and patient may lead to patient discomfort and concerns about whether confidentiality will be maintained. ${ }^{9}$ In addition, in smaller refugee communities, there are fewer translators available (formal or informal), which may lead to a perception that patient confidentially will not be maintained due to the presence of dual relationships. ${ }^{9}$

Financial barriers. Most refugees are provided with coverage through the Interim Federal Health Program (IFHP) when they first arrive to Canada. However, many report difficulties registering for this program, and there is confusion among physicians' offices about who is eligible for the program. ${ }^{9}$ Some refugees have resorted to paying for their health care directly due to being unsure about their coverage. ${ }^{9}$

Systemic barriers. Refugees also cite general barriers to accessing health care that are common to all Canadians, including the availability of services and their cost. ${ }^{6}$ Even in communities which have dedicated refugee health centres, there may be a waiting list to access services. ${ }^{9}$

\section{BEST PRACTICES IN TREATMENT AND MENTAL HEALTH PROMOTION}

Evidence-based practices for supporting refugee mental wellness include the use of psychotherapies, medications, and psychosocial interventions.

Psychotherapies. Most evidence-based approaches to refugee psychotherapy include some combination of supportive counseling and elements of cognitive behavioural therapy (CBT). ${ }^{5}$ Many of these therapies are adapted to be delivered by front-line workers who may be more accessible to patients than psychologists or other 
mental health professionals. ${ }^{5}$

Cognitive behavioural therapy. CBT is a technique that aims to teach patients to re-evaluate external events in a more adaptive way, compared to their previous coping strategies. ${ }^{10} \mathrm{CBT}$ has mainly been trialed for refugees experiencing stress related to their migration, including PTSD. ${ }^{11}$ Overall, CBT appears to be very effective in reducing patients' symptoms of PTSD and other forms of stress. ${ }^{11}$

Specific modules, such as culturally adapted CBT (CA-CBT) have been modified to be more relevant to the unique situations of refugees. CA-CBT differs from traditional CBT in that it includes a modified approach to exposure, emphasizes somatic sensations, and addresses comorbid disorders such as anxiety and anger. ${ }^{10}$

Narrative exposure therapy. Narrative exposure therapy (NET) is another psychotherapeutic treatment that has produced positive results in children and adults diagnosed with PTSD. NET involves the patient chronologically exploring their life, including traumatic events, with the therapist in order to create a life narrative. ${ }^{5,12}$

The actual psychotherapeutic modalities used to treat a specific mental disorder may not be delivered by a physician. However, physicians must still be aware of which therapies are evidencebased when making the decision to refer patients. Unfortunately, many of these psychotherapeutic techniques lack evidence from long-term follow-up. ${ }^{5}$ In addition, patients with more complicated illnesses may not be able to participate actively in psychotherapy, making this an ineffective treatment method for them. ${ }^{5}$

Medication recommendations. There is a lack of research regarding psychiatric medication specifically in refugee populations. ${ }^{5}$ If medication regimens are required to treat mental illness in refugees, physicians generally advise using the same medication regimens that are used in the general population. ${ }^{5}$ Commonly, selective serotonin reuptake inhibitors and tricyclic antidepressants are used to treat depression, PTSD, and anxiety disorders. ${ }^{5}$ However, the affordability of medications may be a barrier given that many refugees cite cost as a reason they are unable to access health care. ${ }^{6}$

Mental health promotion and psychosocial interventions. Refugee mental health may also be affected by the post-displacement environment, and social programs for refugees aim to treat this risk factor. ${ }^{5}$ One group intervention, sociotherapy, has been applied to several refugee populations. ${ }^{5}$ Sociotherapy involves a group discussion about personal and community issues in the presence of a trained facilitator. This intervention aims to increase connections between people and has been shown to improve mental health and community engagement. ${ }^{5}$

In Canada, the Centre for Addiction and Mental Health (CAMH) has laid out 13 best practices for refugee mental health promotion. ${ }^{13}$ These best practices highlight what communities must do in order to create an environment where refugees are able to seek and receive mental health treatment. CAMH recommends that all refugee mental health promotion programs aim to improve protective factors and protective determinants of health against mental illness. ${ }^{13}$ Protective factors include family cohesion, literacy, and adaptability, and protective determinants of health include adequate medical services, housing conditions, and social support. ${ }^{13} \mathrm{CAMH}$ also recommends attempting to reduce mental illness stigma within refugee communities, multidisciplinary collaboration, focusing on resilience, and ensuring that services are culturally appropriate. ${ }^{13}$

\section{ADVOCACY FOR REFUGEE MENTAL HEALTH CARE}

Physicians are well-placed to become advocates for refugee health. This was recently illustrated when the Canadian government made cuts to IFHP coverage in 2012. A group of Canadian physicians formed Canadian Doctors for Refugee Health Care. This advocacy group focused on repealing these cuts, and played a role in the reinstatement of coverage. ${ }^{14}$

Some authors have highlighted gaps in medical education with regards to the care of patients from different cultures. ${ }^{15}$ However, in recent years, medical schools have made efforts to teach their students cultural competency and social accountability. For example, the Association of Faculties of Medicine of Canada is working to ensure medical schools provide teaching about Indigenous health care. ${ }^{16}$ Culturally-competent care also includes trauma-informed care, where care providers are aware of the trauma and hardships faced by their patients. ${ }^{17}$ Recent research has shown that providers lack appropriate training to provide this trauma-informed care, which is another gap in medical education..$^{18}$

In addition to advocating for changes to refugee health care, students and physicians can familiarize themselves with the IFHP. Across Canada, the program is administered by a third party provider. Physicians must register with Medavie Blue Cross and submit all claims for services provided under the IFHP through them. ${ }^{19}$ Some procedures and codes are automatically eligible for payment, however others require pre-approval. ${ }^{19}$ In addition, physicians should be aware that IFHP payments are often slower than Ontario Health Insurance Plan payments and reimbursement may be made at lower rates. ${ }^{9}$

\section{CONCLUSION}

It is clear that the trauma refugees may have faced before settling in Canada provides a significant stressor that contributes to the high rates of mental illness experienced by this population. In addition, once in Canada there are barriers that may prevent refugees from accessing care, leading to unmet health care needs. ${ }^{6}$ However, many refugees also display extraordinary resiliency that can be drawn on when planning mental health outreach and health promotion programs. ${ }^{13}$

\section{REFERENCES}

1. United Nations High Commissioner for Refugees. Figures at a glance: statistical yearbooks [Internet]. 2017 [cited 2018 Dec 5]. Available from: https://www.unhcr.org/figures-at-a-glance.html

2. UN High Commissioner for Refugees. The 1951 convention relating to the status of refugees and its 1967 protocol [Internet]. UNHCR: The UN Refugee Agency; 2011 [cited 2019 Feb 8]. Available from: https:// www.unhcr.org/about-us/background/4ec262df9/1951-conventionrelating-status-refugees-its-1967-protocol.html

3. Government of Canada. Canada: a history of refuge [Internet]. 
Government of Canada. 2018 [cited 2018 Dec 5]. Available from: https://www.canada.ca/en/immigration-refugees-citizenship/ services/refugees/canada-role/timeline.html

4. Puzic S. Record number of refugees admitted to Canada in 2016, highest since 1980. CTV News [Internet]. 2017 Apr 24 [cited 2018 Dec 5]. Available from: https://www.ctvnews.ca/canada/record-numberof-refugees-admitted-to-canada-in-2016-highest-since-1980-1.3382444

5. Silove D, Ventevogel P, Rees S. The contemporary refugee crisis: an overview of mental health challenges. World Psychiatry. 2017 Jun;16(2):130-9. https://doi.org/10.1002/wps.20438

6. Oda A, Tuck A, Agic B, et al. Health care needs and use of health care services among newly arrived Syrian refugees: a cross-sectional study. CMAJ Open. 2017 May;5(2):E354-8. https://doi.org/10.9778/ cmajo.20160170

7. Shannon PJ, Wieling E, Simmelink-McCleary J, et al. Beyond stigma: barriers to discussing mental health in refugee populations. J Loss Trauma. 2015 May;20(3):281-96. https://doi.org/10.1080/15325024.20 $\underline{14.934629}$

8. Ahmed S, Shommu NS, Rumana N, et al. Barriers to access of primary healthcare by immigrant populations in Canada: a literature review. J Immigr Minor Health. 2016 Dec;18(6):1522-40. https://doi. org/10.1007/s10903-015-0276-Z

9. McKeary M, Newbold B. Barriers to care: the challenges for Canadian refugees and their health care providers. J Refug Stud. 2010 Dec;23(4):523-45. https://doi.org/10.1093/jrs/feq038

10. Hinton DE, Rivera EI, Hofmann SG, et al. Adapting CBT for traumatized refugees and ethnic minority patients: examples from culturally adapted CBT (CA-CBT). Transcult Psychiatry. 2012 Apr;49(2):340-65. https://doi.org/10.1177/1363461512441595

11. Murray KE, Davidson GR, Schweitzer RD. Review of refugee mental health interventions following resettlement: best practices and recommendations. A J Orthopsychiatry. 2010;80(4):576-85. https:// doi.org/10.1111/j.1939-0025.2010.01062.x

12. Fazel M. Psychological and psychosocial interventions for refugee children resettled in high-income countries. Epidemiol Psychiatr Sci. 2018 Apr;27(2):117-23. https://doi.org/10.1017/S2045796017000695

13. Centre for Addiction and Mental Health. Best practice guidelines for mental health promotion programs: refugees [Internet]. 2012 [cited 2018 Nov 25]. Available from: https://www.porticonetwork.ca/ documents/1399720/1402901/Refugees/3974e176-69a8-4a5f-843ba40d0a56299c

14. Pottie K, Gruner D, Magwood O. Canada's response to refugees at the primary health care level. Public Health Res Pract. 2018;28(1): e2811803. https://doi.org/10.17061/phrp2811803

15. Chugh U, Dillmann E, Kurtz SM, et al. Multicultural issues in medical curriculum: implications for Canadian physicians. Med Teach. 1993;15(1):83-91. https://doi.org/10.3109/01421599309029015

16. The Association of Faculties of Medicine of Canada. Report on Indigenous health activities [Internet]. The Association of Faculties of Medicine of Canada; 2017 [cited 2018 Nov 25]. Available from: https://www.afmc.ca/sites/default/files/documents/en/AFMC_2017 ReportOnIndigenousHealthActivities_EN.pdf

17. Vukcevic M, Momirovic J, Puric D. Adaptation of Harvard trauma questionnaire for working with refugees and asylum seekers in Serbia. Psihologija. 2016;49(3):277-99. https://doi.org/10.2298/PSI1603277V

18. Wylie L, Van Meyel R, Harder H, et al. Assessing trauma in a transcultural context: challenges in mental health care with immigrants and refugees. Public Health Rev. 2018 Dec;39(1):22. https://doi.org/10.1186/s40985-018-0102-y

19. Medavie Blue Cross. Information handbook for health-care professionals [Internet]. 2016 [cited $2019 \mathrm{Feb} 8$ 8]. Available from: https://docs.medaviebc.ca/providers/guides_info/IFHP-InformationHandbook-for-Health-care-Professionals-April-1-2016.pdf 